\title{
To Effectively Combine the Science and Engineering Teaching in Higher Education from the Scientific Phenomena
}

\section{Deng-Guang YU*, Ding WANG, Shi-You ZHENG, Xian-Ying WANG*, Xiao-Hong CHEN, Hao SUN, Yun TANG and Yi-Yong HE}

\author{
School of Material Science and Engineering, University of Shanghai for Science and Technology, \\ Shanghai 200093, China \\ *Email: ydg017@usst.edu.cn, xianyingwang@usst.edu.cn
}

Keywords: Higher education, Scientific phenomenon, Science courses, Engineering courses, Electrospun nanofibers.

\begin{abstract}
It becomes more and more important for the senior college students to train their capability of connecting theory with practice today. How to provoke the students' interests and effectively nurture their practical ability often puzzle the teachers a lot because that there are only very limited practical resources in the high school. However, the scientific phenomena happen in modern laboratories every day, particularly those can be observed using naked eyes, are good teaching materials. These materials can be used to inspire the students curiosities, and in turn encourage them to do the experiments themselves. Meanwhile, during the processes of experimental practice and writing the experimental reports, the students can review the theoretical knowledge they have learned in their classrooms and it should be easier for them to be imparted of new knowledge. Here, based on the applications of electrospun organic-inorganic hybrid nanofibers in removing dye contaminated water, an excellent example was exhibited for effectively combined the science and engineering teaching.
\end{abstract}

\section{A Phenomenon can String Together a Series of Science and Engineering Courses}

For the senior college students, it is time for them to learn how to gradually utilize the knowledge they have learned in classrooms. For fostering their creativity and capability in linking theory with practice, the selections of teaching materials are very important. Because the practical resources in the university are often very limited, the attempts of some simple scientific researches in laboratories as teaching lessons to them should be an alternative way for solving this problem.

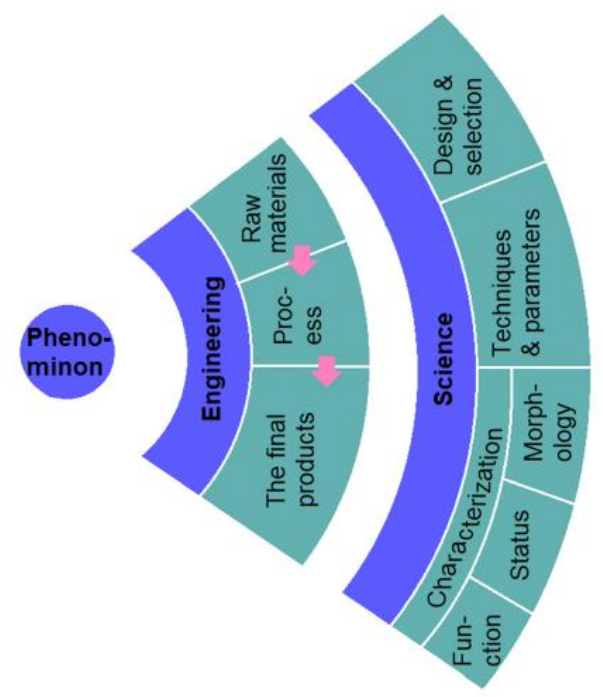

Figure 1. A useful scientific phenomenon is able to string together a series of engineering practices and scientific knowledge. 
The full utilization of the scientific experiments as teaching materials should begin with the scientific phenomena, which not only can be used to provoke the students' great curiosities, promote them to think and try themselves, but also can be exploited to string together a series of sciences and engineering courses (Figure 1). For each scientific phenomenon, it's occurrence has both engineering foundations and also scientific theories to support it. For example, the eye-able phenomenon often appears at the material conversion process with thermal and light effects. All the industrial and laboratory experimental productions have a similar creation procedure, i.e. starting from the selection of raw materials, being subjected to one or a series of treating processes, and the achievement of final desired products. No matter the selection of raw materials or the applications of final products, a series of science courses such as material design and selection, foundations about techniques, and characterizations of new types of materials can be strung together.

\section{An Interesting Phenomenon of Treating Dye Contaminated Water Using Electrospun Nanofibers}

Electrospun fiber is presently a popular one-dimensional nanomaterial, around which many fantastic phenomena happen both in the creation processes and also its functional applications [1-4]. Shown in Figure 2 is about the explorations of electrospun organic-inorganic hybrid nanofibers in removing the dye contaminants from waste water. A sharp contrast of the capabilities of electrospun nanofibers and pure titanium dioxide powders in cleaning the dye contaminated water is obvious, in Figure 2a (compared with the control sample in Figure 2c), the hybrid titanium dioxide-loaded polymeric nanofiber mats were able to convert the blue water into colorless rapidly, while, at the same time period, the pure titanium dioxide nanopowders showed a poorer effecting in treating the blue dye pollution.

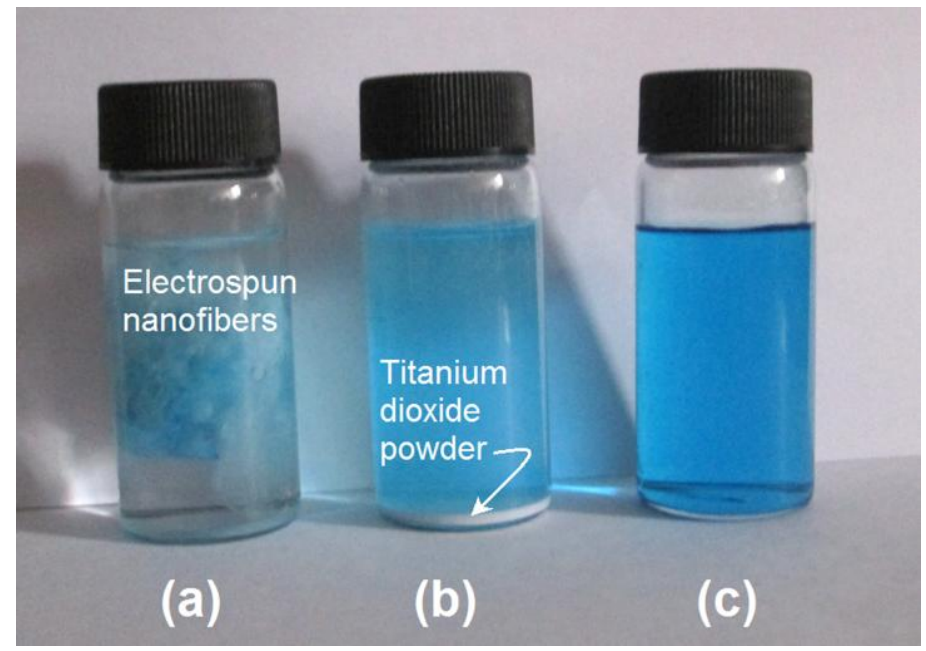

Figure 2. A sharp contrast of the capabilities of electrospun nanofibers and pure titanium dioxide powders in cleaning the dye contaminated water: a) hybrid titanium dioxide-loaded polymeric nanofiber mats; b) titanium dioxide powders; c) the dye-contained sample for control.

Such direct comparisons of the phenomena have drawn a series of questions from the senior college students. These questions can be divided into two groups. One group was about engineering, i.e. the preparation of hybrid nanofibers using electrospinning processes, and their applications in treating polluted water. Another group was about the related scientific knowledge, including applied science and also many fundamental science theories (including physics, chemistry, nanoscience and also polymer sciences). 


\section{The Engineering Practice Foundation for Supporting the Interesting Phenomenon}

The first and foremost question is how to generate the hybrid nanofibers using an electrospinning process, what is the fundamental mechanism for this creation? Shown in Figure 3 is a typical process about preparation of electrospun nanofibers (from material selections to final fiber products). The first step is to select the crude materials (including a filament-forming polymer matrix and the functional ingredient titanium dioxide (Figure 3(1)). The second step is to determine the operational parameters for implementing an electrospinning process, by which nanofibers can be prepared from the nanosuspensions consisting of polymer and titanium dioxide in one or mixed solvents (Figure 3(2)). The third step is the utilization of final hybrid organic-inorganic nanofibers (Figure 3(3) in cleaning dye-contaminated water.

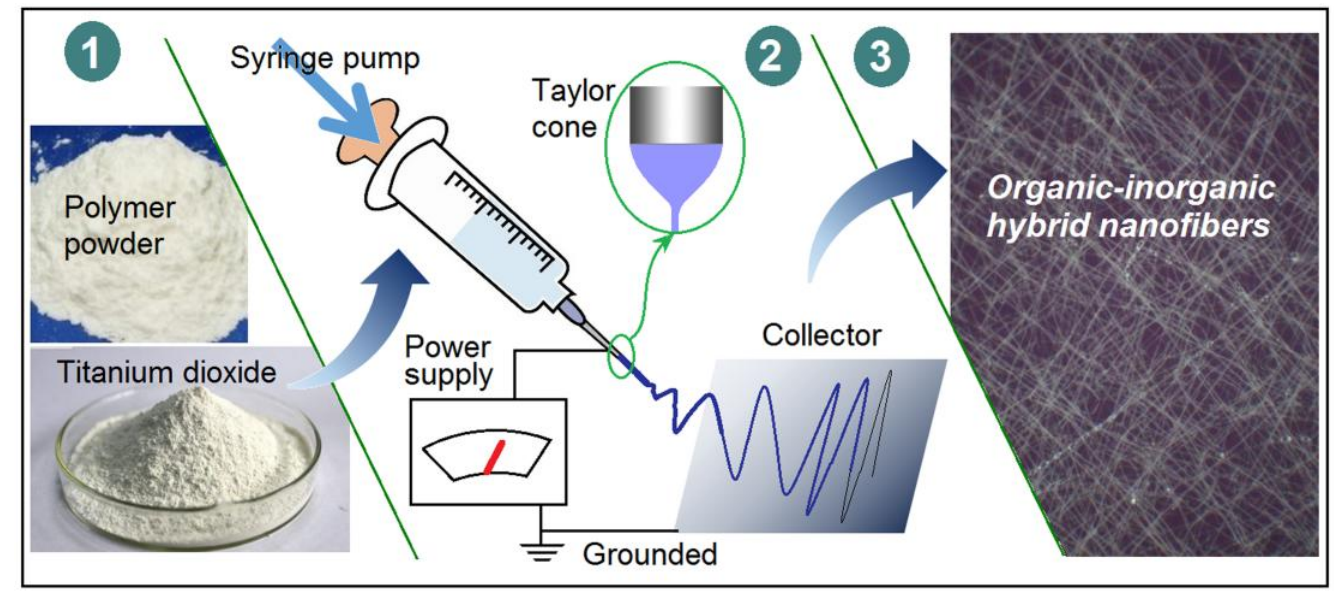

Figure 3. A sequential and interrelated processes that can provide a practice foundation for supporting the interesting phenomenon: (1) the selected crude materials (polymer powders and titanium dioxide powders); (2) a schematic diagram of the single-fluid electrospinning process; (3) an optical observation of the electrospun hybrid organic-inorganic nanofibers (under a magnifications of $20 \times 40)$.

The practice foundations about these processes focus on how to commence a spinning process using the electrostatic force $[5,6]$, during which the formation of a Taylor cone is very important (Figure 3(2)). The Taylor cone is a balance between the strength of the applied high voltage field and the working fluid's physicochemical properties (mainly including surface tension, viscosity and conductivity) [7-9]. A stable Taylor core can ensure a robust and continuous spinning process, and in turn ensure the generation of hybrid nanofibers with high quality. For the senior college students who have experienced basic experiments and some simple engineering practice, the understanding and grasp of electrospinning process should not be a difficult task. Meanwhile, many of their previous experiences about practices and engineering can be combined together here because electrospinning itself is an interdisciplinary phenomenon.

\section{The Theoretical Courses Associated with the Interesting Scientific Phenomenon}

Besides the stringing of practice and experimental experiences, the phenomenon of water treatment can also utilized to promote the students to review the learned knowledge in classrooms and to vigorously study and absorb new basic principles. A schematic diagram about the theoretical courses associated with the interesting phenomenon are shown in Figure 4.

Some typical and basic theory courses include: 1) mathematics and computer science, which can be used to build model to elaborate the adsorption of dye contaminants on the electrospun hybrid nanofibers and their following photocatalytic degradation by titanium dioxide; 2) modern physics, solid state physics and electrohydrodynamics, which can provide a platform for qualitatively describing the electrospinning processes, the solidification of fluid jets and final solid products; 3 ) four major chemistries (organic chemistry, inorganic chemistry, physical chemistry and analytical 
chemistry), applied chemistry, environmental chemistry, polymer science and also nanoscience, which are very important lessons for students majoring in the discipline of "Materials Science and Engineering". Here, the polymer is an organic material, titanium dioxide is an inorganic ingredient, and their nanofibers are a hybrid nanomaterial that find applications in environment. The new type of nanomaterials need to be characterized in terms of morphology, physical status of each components, and functional performances. Thus a series of theoretical courses that the senior college students have learned in their low grades can be effectively lined up from the scientific phenomenon, which can be made full use of training the students' ability of linking knowledge with practice.

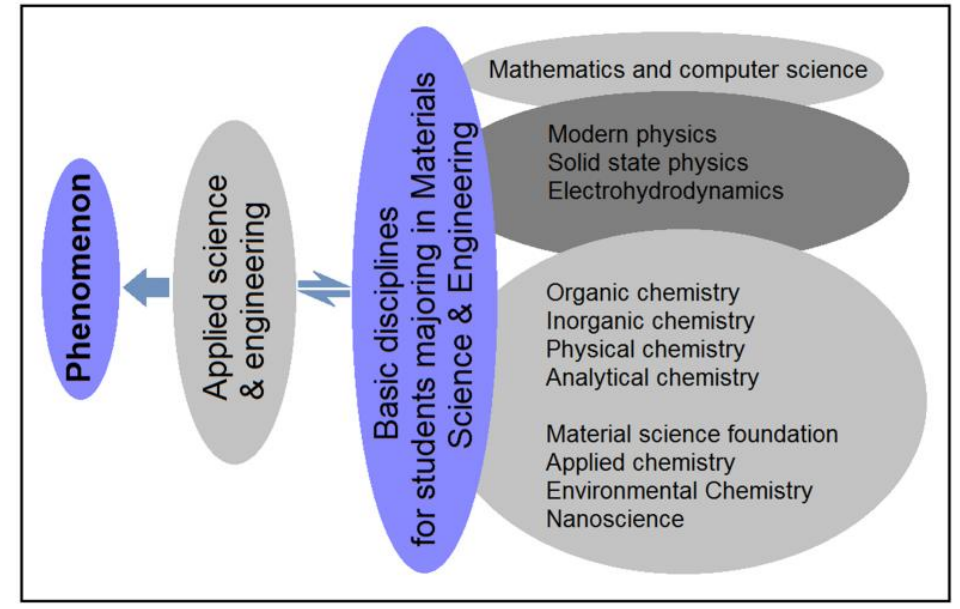

Figure 4. A schematic diagram about the theoretical courses associated with the interesting scientific phenomenon.

\section{Summary}

Starting from an interesting scientific phenomenon, engineering and science teaching can be combined together for fostering the senior college students' capability of connecting theory with practice. The scientific phenomena happened in modern laboratories often have a close relationship with some advanced techniques and the fundamental knowledge, and thus can be effectively utilized as excellent teaching materials. These materials can, on one hand, help the students to improve their ability of linking theory and practice; and on the other hand, to promote the students to review the theoretical knowledge they have learned in their classrooms.

\section{Acknowledgments}

The financial supports from the following projects are appreciated: the National Natural Science Foundation of China (No. 51373101) and the 2016 key project on teachers teaching development in USST (CFTD16001Z), and the College Student Innovation Project of USST (Nos. XJ2016234 and SH2016176).

\section{References}

[1] H.F. Wen, C. Yang, D.G. Yu, X.Y. Li and D.F. Zhang, Electrospun zein nanoribbons for treatment of lead-contained wastewater, Chem. Eng. J., 290 (2016) 263-272.

[2] D.G. Yu, K. White, N. Chatterton, Y. Li, L. Li and X. Wang, Structural lipid nanoparticles self-assembled from electrospun core-shell polymeric nanocomposites, RSC Adv., 5 (2015) 9462-9466.

[3] D.G. Yu, C. Yang, M. Jin, G.R. Williams, H. Zou, X. Wang and S.W. Annie Bligh, Medicated Janus fibers fabricated using a Teflon-coated side-by-side spinneret, Colloid. Surf. B, 138 (2016) 110-116. 
[4] Y.H. Wu, C. Yang, X.Y. Li, J.Y. Zhu, D.G. Yu, Medicated nanofibers fabricated using NaCl solutions as shell fluids in a modified coaxial electrospinning, J. Nanomater., 2016 (2016) 8970213, 12 pages.

[5] Y.H. Wu, H.P. Li, X.X. Shi, J. Wan, Y.F. Liu, D.G. Yu,Effective utilization of the electrostatic repulsion for improved alignment of electrospun nanofibers, J. Nanomater., 2016 (2016) 2067383, 8 pages.

[6] D.G. Yu, X.Y. Li, X. Wang, J.H. Yang, S.W. Annie Bligh and G.R. Williams, Nanofibers fabricated using triaxial electrospinning as zero order drug delivery systems, ACS Appl. Mater. Interf., 7 (2015) 18891-18897.

[7] C. Yang, D.G. Yu, D. Pan, X.K. Liu, X. Wang, S.W. A. Bligh and G. R. Williams, Electrospun $\mathrm{pH}$-sensitive core-shell polymer nanocomposites fabricated using a tri-axial processes, Acta Biomater., 35 (2016) 77-86.

[8] F. Xu, Y. Xu and D.G. Yu, Zero-order controlled release nanofibers fabricated using coaxial electrospinning with polymer dilute solution as a sheath fluid, J. University of Shanghai for Science and Technology, 37 (2015) 165-168.

[9] J. Yan, Y.H. Wu, D.G. Yu, G.R. Williams, S.M. Huang, W. Tao and J.Y. Sun, Electrospun acid-base pair solid dispersions of quercetin, RSC Adv., 4 (2014) 58265 - 58271. 